\title{
A Fabula Model for Emergent Narrative
}

\author{
Ivo Swartjes and Mariët Theune \\ University of Twente, Human Media Interaction group \\ Enschede, The Netherlands \\ \{i.m.t.swartjes, m.theune\}@ewi.utwente.nl
}

\begin{abstract}
In this paper we present our continuing efforts to generate narrative using a character-centric approach. In particular we discuss the advantages of explicitly representing the emergent event sequence in order to be able to exert influence on it and generate stories that 'retell' the emergent narrative. Based on a narrative distinction between fabula, plot and presentation, we make a first step by presenting a model based on story comprehension that can capture the fabula, and show how it can be used for the automatic creation of stories.
\end{abstract}

\section{Introduction}

In the Virtual Storyteller project [1] we investigate how stories can emerge through simulation of a virtual world inhabited by virtual characters, an approach that is often referred to as Emergent Narrative [2. We are currently investigating the possibility to explicitly represent narrative based on the emerging event sequence, so that it can feed a story generation process. We believe that such a formal representation will allow the system not only to express the event sequence that it produces in e.g. natural language, but also to comprehend, and as such, manipulate the event sequence. For this representation, a narratological distinction is made between three layers of information that together constitute narrative. Mieke Bal calls these layers fabula, story and narrative text [3]. The fabula consists of a series of logically and chronologically related events that are caused or experienced by characters in a story world. A story is a fabula that is looked at from a certain angle. A narrative text is an expression of the story in language signs (e.g. words, pictures). Our distinction is similar but more aimed at formalization:

1. The fabula layer is an account of what happens in the story world and why. It is a causal network of all events that took place in the story world.

2. The plot layer is based on a relevant selection of the fabula layer that forms a consistent and coherent whole, adhering to an Aristotelian understanding of these concepts similar to Sgouros [4. One can say that many plots coexist within one fabula.

3. The presentation layer represents the information needed for the actual presentation of the plot in a certain medium. The medium of choice for the Virtual Storyteller is natural language, both written [5] and spoken [6]. In the future, we also intend to investigate graphical presentations. 
In this paper we focus on the fabula layer, how it can be modeled and what it can be used for. The paper is structured as follows. In section 2 , we discuss why we need an explicit fabula structure for story generation, and how we intend to use it. In section 3 we present our model of fabula structure and show how it can be generated. Guided by an example, section 4 shows how such a structure can be transformed into a written story. Finally, in Section [5] we provide some conclusions and discuss how we intend to use the fabula model in the future to support plot guidance and creativity.

\section{The Need for a Formal Narrative Structure}

Our approach to automated story generation is to first generate a fabula layer, then apply selections and transformations in order to form plot and presentation layers. The fabula layer is generated by observing what happens in a Multi-Agent simulation where autonomous, intelligent Character Agents are situated within a virtual story world in which they pursue their goals [1. A risk factor for such an approach is the fact that an interesting plot is not guaranteed. Characters follow their individual goals which are different from the goals of the plot. As a consequence, the stories produced by such an approach may not be very interesting. Therefore, a Plot Agent (similar to the Drama Manager introduced in the OZ project [7]) exerts influence on the emerging event sequence, motivated from a goal to stimulate the occurrence of 'interesting things' from a plot point of view. A prerequisite for the pursuit of plot goals is that the system has some understanding of the plots that are generated. Developing a model of fabula contributes to this by making the causal and temporal relationships between fabula elements explicit, allowing them to be analyzed for 'plot potential'. The system does not commit to any particular plot during the simulation; all story world events, relevant for a plot or not, are captured in the fabula.

Generating stories like this has many advantages. First, consistency and coherence of the fabula underlying any generated story can be ensured by the simulation and is not a plot concern. Second, by making the fabula explicit we have an objective account of events at our disposal which is independent of stylistic concerns such as viewpoints, author opinions, time lapses and couleur locale, and therefore has the potential to be shaped into any form of presentation desired. This makes it possible to tell different stories based on the same fabula. It also makes plot generation independent of presentation; the same plot can form the input for the production of a narrative text, an animation or picture story.

Much research has focused on understanding and formalizing event structures appearing in stories, from both the perspective of narratology (e.g., Propp's seminal work [8] ), and story comprehension (e.g., 9] ). Given the advantages of having an explicit narrative structure for story generation, it is surprising that - as also noted by [10] and 11] - story generation systems often lack a formalization of such structures. This was also the case for previous versions of the Virtual Storyteller, where the lack of a formal fabula representation resulted in a rather boring, literal account of the events occurring in our story world [1]. When representations 
are used in other systems, they are often rather ad-hoc (e.g., the representation used in MINSTREL [12]) or biased towards action planning (e.g., the definition of fabula in the story generation system Fabulist [13]).

A recent attempt in formalizing drama structures is the Drammar ontology 10, a high-level ontology for the representation of drama on which our fabula structure can be said to elaborate. The ontology considers drama on two levels: the actional and the directional level, comparable to our idea of fabula and plot layers. Another recent attempt at formalizing fabula, albeit more focused on annotation, is the OntoMedia project [11].

\section{A Formal Model of Fabula}

We base our fabula model on story comprehension research, since we believe that the use of story comprehension models brings the way the story generation system understands the stories it develops closer to the way humans think about them. In [14, Trabasso et al. presented a story comprehension model, which was later applied to experiments in the narration of picture stories [15. Children and adults were asked to narrate the events that they inferred to be happening in a sequence of pictures, and the clauses that these children used were categorized using the model to expose the underlying narrative structure. This revealed three stages in the development of story comprehension. Very young children comprehend a story as a sequence of isolated states and actions, whereas at a later age, they can identify a temporal ordering 11 Eventually, children learn to identify and express causal relationships between goals, actions and outcomes, developing into a full hierarchical ordering of episodes that comprise coherent stories.

The result of Trabasso's research is the General Transition Network (GTN) model, which forms the basis for our fabula structure for story generation. The GTN model identifies a causal network of six story elements (Setting, Event, Internal Response, Goal, Attempt and Outcome) connected by causal relationships: physical $(\phi)$ and psychological $(\psi)$ causality, motivation $(m)$ and enablement $(e)$. These story elements and their relationships form an implicit hierarchical structure of one or more episodes, which together form a story. The basis for each episode is formed by a causally related Goal, Attempt, and Outcome. Goals set expectations, Outcomes affirm or deny them.

The GTN model is a model of story analysis, which is not useful for story generation unless we succeed to formalize the way in which the different elements and their connections can be created, and subsequently narrated. We propose a model that is based on the GTN model but differs in important aspects. One of the main differences with the GTN model is that the fabula model we propose attempts to capture the fabula in a single (objective) network, whereas in the GTN model a separate network is constructed from the (subjective) viewpoint of

${ }^{1}$ This can be witnessed when these children tell about their experiences: "And then this happened and then that happened and then...". A similar, simple temporal ordering can be seen in stories generated by previous versions of the Virtual Storyteller and other generation systems. 
each character in the story. In the GTN model, something that is an Action for one character can be an Event for another. Our model, however, is independent of viewpoints and therefore offers a global perspective on the fabula.

The fabula model we propose can be seen in Figure 1, showing all possible causal relations between fabula elements. Networks generated using this model do not form a complete account of everything that happened in the course of the story, but only of those fabula elements that have either a cause or an effect on other elements. Unlike Trabasso, we leave the Setting of the story (characters, locations) out of this model. We consider it to be the background against which the story unfolds. The fabula elements are however linked to representations of the characters and objects in the story world via their arguments (e.g., agens and patiens of an Action). At a later point in the story generation process, i.e., when forming the plot or presentation layer, decisions can be made which parts of the setting are conveyed.

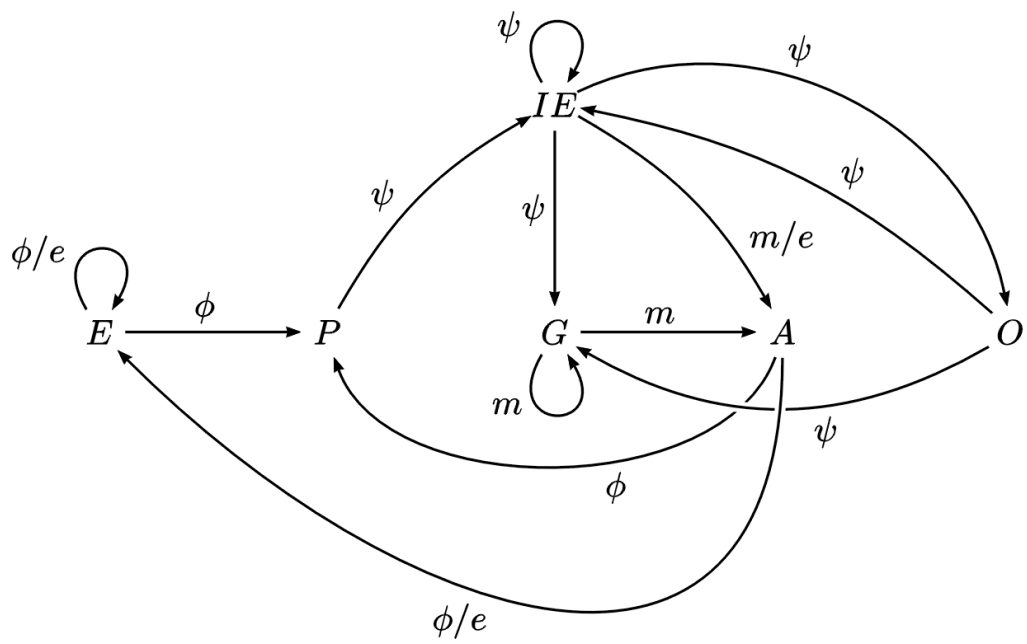

Fig. 1. Fabula model for story generation. The arrows represent possible causal relationships between the elements of the fabula.

\subsection{Fabula Elements}

Our fabula model defines causal relationships between six types of elements: Goals, Actions, Outcomes, Events, Perceptions and Internal Elements. Before we discuss these causal relationships, let us first take a look at the elements themselves, and discuss their differences with the GTN model.

Goal $(G)$. A Goal is the main drive for a character to act. We adopt Trabasso's definition used in the GTN model; a Goal in this context describes a desire to attain, maintain, leave or avoid certain states, activities or objects [14].

Action $(A)$. The term Action is used to indicate a goal-driven, intentional world change brought about by a character. The GTN model uses the term 
Attempt, which from a planning perspective can be seen as a series of Actions that constitute a plan. We use Actions directly since the individual Actions in an Attempt can have separate effects (e.g., an Action can be perceived or cause an Event).

Outcome $(O)$. Every Goal has an Outcome. In the GTN model this Outcome is the real world result of an Attempt. In our fabula model it is a mental concept: when a character believes that its Goal is fulfilled, the Goal has a positive Outcome, but if the character believes that the performed Actions did not succeed in fulfilling the Goal, the Outcome is negative. The Outcome is thus closely tied to the world as the character believes it to be, not necessarily to the world as it actually is

Event $(E)$. From the personal viewpoint of the GTN model, an Event is anything that evokes a response for a character, and in this sense an action of one character can be an event for another. From the global perspective of our fabula model, however, an Event is defined as any change in the world that is not the direct and planned result of any character action, e.g. a tree that falls down, or a twig that breaks.

Perception $(P)$. Perceptions are understandably lacking in the GTN model since the presence of an element in the character's personal network implies that it has been perceived by that character. However, when adopting a global fabula perspective, the explicit notion of Perception is important because the Character Agents do not necessarily perceive all that happens in the story world.

Internal Element $(I E)$. Anything that happens within a character, such as cognitions, emotions, feelings and beliefs, is considered to be an Internal Element. We prefer to use the term 'Internal Element' instead of 'Internal Response' as used in the GTN model, because the word 'response' suggests that there is a cause even though there isn't always one, at least not at the level of abstraction we intend to use 3

The model has been implemented as an OWL ontology; the aforementioned elements are only the top elements of a more extensive subsumption hierarchy. For instance, Goal subsumes AttainGoal and AvoidGoal, Internal Element subsumes BeliefElement and Emotion, and Perception subsumes See and Hear. Furthermore, properties are available for each element that allow for the expression of knowledge about which Character Agent originated or experienced the fabula element, and the time at which the element occurred (in terms of discrete, virtual time steps in the story world). This enables a temporal ordering of the elements.

\footnotetext{
$\overline{2}$ This enables some interesting dramatic situations. For instance in the story of Romeo and Juliet, Juliet pretends to be dead. Romeo however thinks Juliet is really dead, which is a very negative Outcome for his Goal to be together with her.

${ }^{3}$ Consider for instance the Internal Element "hunger". This element could be seen as caused by complicated biophysical processes, but this seems overly specific for most story generation domains.
} 


\subsection{Causally Connecting the Fabula Elements}

Following [14, we distinguish four types of causality that are used to connect the fabula elements: physical causality, psychological causality, motivation and enablement. These types differ in causal strength [16, physical causality being the strongest, followed by motivation, psychological causation and enablement. This difference in causal strength can be reflected in the generated presentation, i.e., stronger causal relationships might need less explicit wording. Note that these causal relationships only reflect what is happening in the simulated story world; they have no generative power themselves.

We realize that any attempt to formalize a layer of causality is unavoidably connected to assumptions about the generative processes of the underlying narrative simulation. We will therefore make these assumptions explicit where necessary. Let's consider the possible causal relationships between the six elements of the fabula, from strong to weak.

Physical causality $(\phi)$. When an Event or Action happens in the story world and causes something else to happen, this causality is physical. This is the strongest form of causality and might not need to be made explicit in the presentation of the story 4

1. Actions physically cause Events. For instance, when a knight stabs a dragon with a sword, this can physically cause the dragon to die. This can also be used as a way to model the occurrence of non-standard results: when the knight crosses a small bridge, it can cause the bridge to break.

2. Events physically cause other Events. For instance, a tree that falls on a bridge causes the bridge to collapse. Or Sleeping Beauty pricking herself on the spinning wheel (an unintended action, thus an Event) causes her to fall asleep.

3. Events and Actions physically cause Perceptions. When a character perceives either the Events or Actions themselves, or their results, such Perceptions are caused by the Events and Actions.

Motivation $(\boldsymbol{m})$. Motivation $(m)$ is an intentional causality, originating within the Character Agents' minds. Wanting to kill the monster can motivate the knight to stab it with a sword. Motivation relates to Riedl's definition of character believability [13, i.e., that the events in a story are reasonably motivated by the beliefs, desires and goals of the characters that participate in the events.

1. Goals motivate other Goals. A Goal $G_{1}$ motivates another Goal $G_{2}$ when $G_{2}$ is a subgoal of $G_{1}$. For instance, the Goal of a knight to kill a dragon could motivate a subgoal of finding the dragon.

\footnotetext{
${ }^{4}$ In fact, this causality is often so strong that it is difficult for a reader to not infer it, even when unstated. Take for instance the two Actions John fired his gun and Peter died. Most readers presented with these successive actions would infer that John shot Peter, but this is not stated explicitly and other explanations are very well possible.
} 
2. Goals motivate Actions. Using a planning algorithm ensures that the planned Actions are driven by one or more (motivating) Goals. Each Action from a generated plan will be motivated by the Goal for which the plan was made.

3. Internal Elements motivate Actions. Actions that are causally connected to Internal Elements are reflex-like Actions like crying and screaming, that are directly caused by an Internal Element and not by a strategic attempt to fulfill a Goal.

Psychological causality $(\boldsymbol{\psi})$. Psychological causality $(\psi)$ takes place within the mind of the characters. For instance, if a knight believes that a dragon is going to kill him, this psychologically causes fear within him. It is causality on the level of the cognitive processes of the Character agents. The difference with motivation is that psychological causality is not intentional. A causal chain of event appraisal, as described in 1, can be identified where perceptions lead to Goals: (1) Perceptions psychologically cause Internal Elements, i.e., beliefs; (2) Internal Elements (beliefs) psychologically cause other Internal Elements (emotions); (3) Internal Elements psychologically cause Goals. The Character Agents need a way to determine Goals based on their beliefs, desires and emotions, which are then seen as psychologically causing the Goal.

Psychological causality also applies to Outcomes, since the Outcome is a mental concept. Internal Elements psychologically cause Outcomes when a Character Agent believes that a plan for his Goal failed or succeeded: a positive Outcome when a plan succeeds and a negative one when the plan failed. When a character stops wanting to achieve the Goal, this will lead to a neutral Outcome. These Outcomes can in turn psychologically cause Goals or Internal Elements. Positive Outcomes lead to positive emotions; negative Outcomes lead to negative emotions and possibly the reinstatement of the failed Goal.

Enablement $(e)$. Enablement $(e)$ is the weakest form of causality. If a fabula element A enables another fabula element B, this means that B is possible because of $\mathrm{A}$, but no more than that. An obvious formalization of enablement is that the effects of A satisfy preconditions of B. In the case of an Internal Element enabling an Action, the requirement would be that the Internal Element is a belief and the contents of that belief satisfy preconditions of the Action.

Note that the model does not include Actions enabling other Actions; because Actions are consciously planned by the characters (except if they are directly caused by emotions, without any deliberation) they can only be enabled by beliefs. E.g., killing a rabbit does not enable eating the rabbit until the character sees and believes that the rabbit is indeed dead. Since Events are not planned, this restriction does not apply to them. Note also that as a consequence, Events can only occur if their preconditions are truly met by the Story World, whereas a Character may be mistaken about meeting the preconditions of an Action. 


\section{Example: Generating a Story from a Fabula}

One of the main reasons for making the fabula structure explicit is to be able to generate coherent stories based on it. In this section we show how a (written) story can be generated from a simple fabula. As an example we use the fabula of a fragment from "Princess Lovely", a story written by Katri Oinonen. The story is about Princess Lovely, who lives alone with her father in a big castle. She wants to play with some children she sees outside, but her father, King Mikura, does not allow it. Instead he gives her a little lamb to play with. Princess Lovely loves the lamb very much, but one day it runs away. Lovely searches for the lamb, only to discover that the children she wanted to play with have slaughtered the lamb because they were hungry. Figure 2 shows the elements of the fabula network we have (manually) reconstructed for the final part of the story, starting from the moment that Princess Lovely hears that the lamb has run away. Figure 3 shows this fabula network 5

From this fabula, first we select those elements we want to include in the plot. Presenting the entire fabula is possible, but, as argued earlier, this generally does not result in a very interesting story. An example method to form a plot layer based on the fabula layer is to select one goal to form the core of the plot (Sgouros calls this the Storyline Goal [4), necessarily turning the character having the goal into the protagonist. The selection process can then be based on this goal by including everything that is affiliated to this goal, nothing more, nothing less 6 Another, simpler way of creating a plot from a fabula is to focus on one character, and include only elements directly pertaining to this character in the plot structure. (A more advanced selection method is presented by [17, who describe how to construct a plot from a fabula so as to maximize suspense.) In our example, we choose Princess Lovely as the protagonist and select the bold-faced elements from Figure 2 for inclusion in the plot structure. Note that besides Lovely's Goals, Actions, etc. we also include one Action performed by the children (slaughtering the lamb) in the plot, because it directly causes one of Lovely's Perceptions.

The resulting plot structure forms the input for the Narrator component of the Virtual Storyteller, which generates a natural language text in three steps. The first step is the creation of a 'document plan': a binary branching tree containing selected elements from the plot structure, connected by rhetorical relations that are suitable for expression in natural language. Constructing the document plan involves removing those plot elements that will not be explicitly expressed in the story, e.g., beliefs caused by Perceptions, which are considered to be inferable by the reader. It also involves adding new elements that represent background information about the story world or properties of the characters, e.g., Lovely

\footnotetext{
5 To save space, we have only included elements pertaining to Lovely and the children, not other characters such as King Mikura. As a further simplification, we have left out some elements that are not essential for understanding the course of the fabula, e.g., many other Perceptions that have occurred as a consequence of Actions and Events.

${ }^{6}$ It remains to be investigated what it means for fabula elements to be affiliated with a goal.
} 


\begin{tabular}{|c|}
$I E_{c(1)}:$ The children are hungry. \\
$G_{c(1)}:$ The children want to stop being hungry. \\
$P_{L(1)}:$ Lovely hears that the lamb has fled. \\
$I E_{L(1)}:$ Lovely believes the lamb is gone. \\
$I E_{L(2)}:$ Lovely is sad. \\
$G_{L(1)}:$ Lovely wants the lamb back. \\
$G_{L(2)}:$ Lovely wants to find the lamb. \\
$A_{L}:$ Lovely runs out of the castle. \\
$E_{1}:$ The lamb appears on the children's location. \\
$P_{c(1)}:$ The children see the lamb. \\
$I E_{c(2)}:$ The children believe the lamb is there. \\
$G_{c(2)}:$ The children want to eat the lamb. \\
$A_{c(1)}:$ The children slaughter the lamb. \\
$P_{L(2)}:$ Lovely sees the lamb. \\
$I E_{L(3)}:$ Lovely believes the lamb is there. \\
$I E_{L(4)}:$ Lovely believes the lamb is dead. \\
$O_{L(2)}^{+}:$(Lovely has found the lamb) \\
$O_{L(1)}^{-}:$(Lovely cannot have the lamb back) \\
$I E_{L(5)}:$ Lovely is sad and hurt. \\
$A_{c(2)}:$ The children cook the lamb. \\
$A_{c(2)}:$ The children eat the lamb. \\
$P_{c(2)}:$ The lamb is eaten. \\
$I E_{c(4)}:$ The children believe they have eaten the lamb. \\
$O_{c(2)}^{+}:$(The children have eaten the lamb) \\
$I E_{c(3)}:$ The children are not hungry. \\
$O_{c(1)}^{+}:$(The children are no longer hungry) \\
\end{tabular}

Fig. 2. Fabula elements of a selection of the Princess Lovely story. Bold-faced elements are included in the plot structure underlying the presentation in Figure 4.

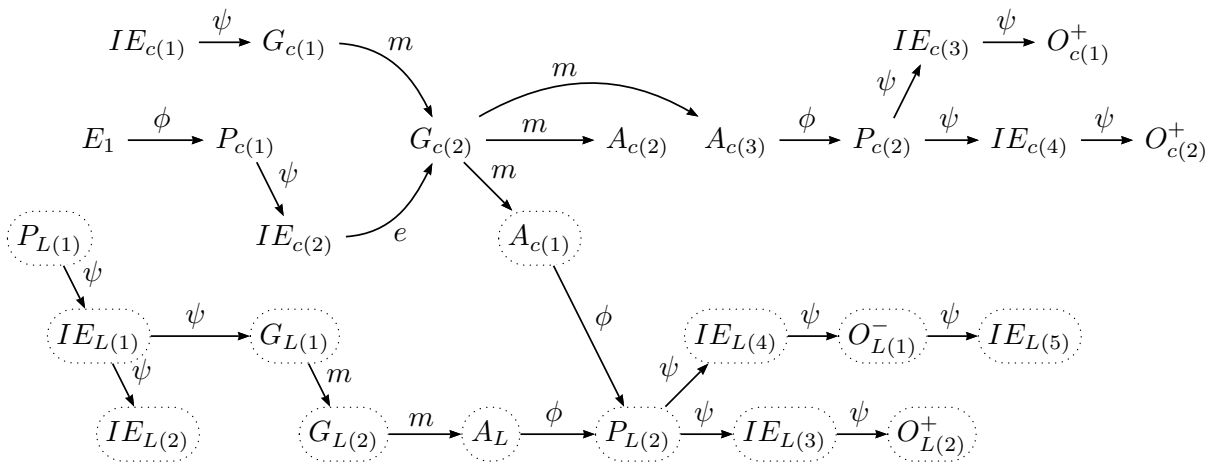

Fig. 3. Network showing the fabula elements of Figure 2 and their causal relationships. From left to right, the elements are chronologically ordered. Encircled elements are included in the plot structure underlying the text in Figure 4 . 
Because Princess Lovely heard that the lamb had fled, she was sad.
She wanted the lamb back, so she wanted to find it and ran out of the
castle. Meanwhile, the hungry children slaughtered the lamb. Because
Lovely saw the dead lamb, she was sad and hurt.

Fig. 4. Example outcome of generation for the Princess Lovely story

being a princess and the lamb being dead after having been slaughtered. In the second step, the leaf nodes (plot elements) of the document plan are replaced with abstract sentence structures called Dependency Trees. In the third step, these Dependency Trees are combined to form complex sentences, in which the clauses are connected with appropriate cue words (because, then, but, ...) to express the rhetorical relations between them. Recurring elements in these complex sentences are deleted where possible; e.g., Lovely cried and Lovely screamed becomes Lovely cried and screamed. Next, appropriate referring expressions such as pronouns are generated for all references to characters and objects that have not been deleted, and the abstract sentence structures are made concrete by applying rules for word order and morphology. A possible result of all these processes for our input plot structure is shown in Figure 4 (translated into English).

Not all parts of the generation process described here have been implemented; most notably we do not have automated conversion from fabula to plot yet. Transforming plot information into a presentation involves making a further selection of what is to be expressed explicitly and what is to be left to be inferred by the reader, but also involves processes like adding relevant background information, applying stylistic ordering and adding couleur locale. The Narrator component, however, has been fully implemented. The construction of complex sentences with appropriate cue words by the Narrator is discussed in detail in [5]; all other parts of the Narrator are described in [18].

\section{Conclusions and Future Work}

In this paper, we have discussed a model that can be used to capture the fabula of Emergent Narrative. We have described the fundamental elements of the model and four types of causal relationships between these elements, as identified by story comprehension theory. Furthermore, we have shown how these causal relationships can arise using a character-based approach to automated story generation.

Due to our use of the formal fabula model, in combination with a sophisticated Narration component that can make the most of the information specified in the fabula, we can generate stories that are more coherent than those generated by previous versions of the Virtual Storyteller, although by no means of the quality achievable by a human author. In contrast to systems that only produce a list or plan sequence of actions or events, expressed by means of simple sentences (e.g., 13 17 19]), the Virtual Storyteller also mentions the underlying emotions and goals, and expresses them in fluent text using complex sentences connected by suitable cue words. 
Further work on improving the Narrator is still necessary. More importantly, we still need good methods to transform fabula structures into plot structures. We will use the work of [4] and [17] as a starting point for this.

Finally, we would like to continue investigating the use of our fabula model for guiding plot development using creative problem solving. Fabula structures as identified in this paper can be used to represent cases and problems as input for Case Based Reasoning (CBR), similar to MINSTREL 12. CBR can support the Plot Agent in making authorial decisions to guide the fabula development on different levels of control [20]. On the environmental level, Events can be generated to influence the plans of characters, perceptions of the characters can be influenced, and changes in the setting of the story world can be made in the course of the generation (initial state revision, as in 21]). On the behavioral/motor skill level, the characters can be directed by suggesting Goals or Actions? Such problems can be expressed using partially uninstantiated fabula fragments. Based on a case base of fabula fragments, novel solutions can be found using creativity heuristics. For instance, the problem "what Event can be caused by the children eating the lamb?" can be solved using a stored fabula fragment expressing for instance that Snow White fell asleep after eating an apple, leading to the creative solution that eating the lamb causes the children to fall asleep. To this end, we are currently in the process of defining problem space requirements and creativity heuristics. We believe that the Character Agents can also benefit from CBR by using it to aid their deliberation processes (i.e., to interpret Perceptions or to reason about possible consequences of their planned Actions).

\section{References}

1. Theune, M., Rensen, S., op den Akker, R., Heylen, D., Nijholt, A.: Emotional characters for automatic plot creation. In Göbel, S., et al., eds.: Technologies for Interactive Digital Storytelling and Entertainment (TIDSE). Lecture Notes in Computer Science 3105, Springer-Verlag (2004) 95-100

2. Louchart, S., Aylett, R.: Narrative theory and emergent interactive narrative. Int. J. Continuing Engineering Education and Lifelong Learning 14 (2004) 506-518

3. Bal, M.: Narratology: Introduction to the Theory of Narrative. 2nd edn. University of Toronto Press (1997)

4. Sgouros, N.M.: Dynamic generation, management and resolution of interactive plots. Artificial Intelligence 107 (1998) 29-62

5. Theune, M., Hielkema, F., Hendriks, P.: Performing aggregation and ellipsis using discourse structures. Research on Language and Computation, Special Issue on Cross-Modular Approaches to Ellipsis (2007) to appear.

6. Theune, M., Meijs, K., Heylen, D., Ordelman, R.: Generating expressive speech for storytelling applications. IEEE Transactions on Audio, Speech and Language Processing 14 (2006) 1137-1144

\footnotetext{
${ }^{7}$ We consider suggestion to be a weak form of prescriptive control aimed at keeping the Character Agents autonomous, basically telling them: make your own goals and plan your own actions, but if any of those contain the suggested goals or actions, give preference to intentions that contain the suggestions.
} 
7. Kelso, M.T., Weyhrauch, P., Bates, J.: Dramatic presence. PRESENCE: The Journal of Teleoperators and Virtual Environments 2 (1993) 1-15

8. Propp, V.: De morfologie van het toversprookje: vormleer van een genre. Spectrum, Utrecht (1997)

9. Van Dijk, T.A.: Story comprehension: An introduction. Poetics 9 (1980) 1-21

10. Damiano, R., Lombardo, V., Pizzo, A.: Formal encoding of drama ontology. Lecture Notes in Computer Science 3805 (2005) 95-104

11. Tuffield, M.M., Millard, D.E., Shadbolt, N.R.: Ontological approaches to modelling narrative. In: Proceedings of 2nd AKT DTA Symposium. (2006)

12. Turner, S.R.: The creative process: a computer model of storytelling. Lawrence Erlbaum Associates, Hillsdale, NJ (1994)

13. Riedl, M.O.: Narrative Generation: Balancing Plot and Character. PhD thesis, North Carolina State University (2004)

14. Trabasso, T., Van den Broek, P., Suh, S.Y.: Logical necessity and transitivity of causal relations in stories. Discourse Processes 12 (1989) 1-25

15. Trabasso, T., Nickels, M.: The development of goal plans of action in the narration of a picture story. Discourse Processes 15 (1992) 249-275

16. Tapiero, I., Van den Broek, P., Quintana, M.P.: The mental representation of narrative texts as networks: The role of necessity and sufficiency in the detection of different types of causal relations. Discourse Processes 34 (2002) 237-258

17. Cheong, Y.G., Young, R.M.: A computational model of narrative generation for suspense. In: Proceedings of the Twenty-First National Conference on Artificial Intelligence (AAAI06). (2006)

18. Slabbers, N.: Narration for virtual storytelling. Master's thesis, University of Twente (2006)

19. Meehan, J.: TALE-SPIN. In Schank, R., Riesbeck, K., eds.: Inside computer understanding - five programs plus miniatures. Lawrence Erlbaum Associates (1981) $197-226$

20. Blumberg, B., Gaylean, T.: Multi-level control for animated autonomous agents: Do the right thing... oh, not that... In Trappl, R., Petta, P., eds.: Creating Personalities for Synthetic Actors: Towards Autonomous Personality Agents. Springer-Verlag (1997) 74-82

21. Riedl, M.O., Young, R.M.: Open-world planning for story generation. In: International Joint Conference on Artificial Intelligence (IJCAI). (2005) 1719-1720 